\title{
Nasal Cavity Masses: Clinico-Radiologic Collaborations, Differential Diagnosis by Special Clues
}

\author{
Duzgun Yildirim ${ }^{1}$, Omer Saglam², Berk Gurpinar², Turan Ilica ${ }^{3}$ \\ ${ }^{1}$ Department of Radiology, Iskenderun Military Hospital, Hatay, Turkey \\ ${ }^{2}$ Department of Head and Neck Surgery, Kasimpasa Military Hospital, Istanbul, Turkey \\ ${ }^{3}$ Department of Radiology, Gulhane Military Medical Academy, Ankara, Turkey \\ Email: yildirimduzgun@yahoo.com
}

Received November 18, 2011; revised December 30, 2011; accepted January 13, 2012

\begin{abstract}
Purpose: Nasal cavity may contain wide variety of masses within, that differs this organ from the rest of the body. Primary nasal cavity masses consist of $0.2 \%-0.8 \%$ of all malignancies. This paper aims to emphasize the main characteristics of different nasal cavity masses on cross-sectional images which may cause symptoms varying from simple nasal obstruction to metastatic invasion. We tried to solve the diagnostic bias by focusing on the special clues with the aid of the striking images caused by the same appearence of nasal cavity masses on cross-sectional radiologic images. Materials and Method: 66 retrospective dataset of patients (male: 35, female: 31, mean age: 43 years) were reviewed by the cross-sectional images. All cases had nasal passage obstruction and all cases had previously undergone maxillofacial imaging (computerized tomography, CT $(n=43)$; magnetic resonance imaging, MRI $(n=21)$; positron emmision tomography, PET/CT $(n=2))$. Results: Totally, 48 benign and 18 malignant cases which have distinct pathologies were reviewed. All the lesions occupying space through the nasal cavity were demonstrated on cross-sectional images. With the typical cross-sectional images, an algoritm was made to help the differential diagnosis and presented as a scheme to presume the most feasible diagnosis. Conclusion: Sinunasal masses may have the worst prognosis on late diagnosis because of the probability of early invasion of the basicranial structures or cranial nerves. Verification of the neoplasm by the specific cross-sectional images, either benign or malignant, could be done at once.
\end{abstract}

Keywords: Nasal Cavity; Neoplasms; Imaging; Differential Diagnosis

\section{Introduction}

Nasal cavity contains different kinds of tissues such as the epithelial (squamous, neuroendocrine, olfactory) and the mesenchimal (bone, cartilage, muscle, vascular) ones and all of these may carry the risk for a variety of tumoral differentiation. Despite the wide range of diversity, the incidence of the nasal tumors are as low as $1 / 100.000$ [1]. Primary nasal malignancies consist of $0.2 \%-0.8 \%$ of all the malignant tumors and $3.6 \%$ of the malignant upper airway tumors [1].

CT (computerized tomography) and MRI (magnetic resonance imaging) were useful in evaluating various kind of the nasal cavity masses. They may differentiate the contents, contrast enhancement patterns of the lesions and localize the tumor, thus these cross-sectional imaging modalities are being used frequently to differentiate the nasal cavity masses [1]. Additionally, PET/CT, which is consisted by fusion of both the PET (positron emission tomography) and CT systems, has the advantage of providing functional assessment additional to lesion deter- mination [1].

In this study our primary goal was to mention the common features of the benign and malignant nasal tumors that usually give symptoms such as nasal congestion, blockage, rhinorrheae, headache, proptosis, trismus, cranial neuropathy; whereas the radiologic examination of the cross-sectional images of various types of nasal tumors that mostly block the airway. We also briefly stated the main clinic and surgical approaches to those cases and gave a brief discussion of the literature.

\section{Materials and Methods}

66 cases (male: 35 , female: 31 , mean age: 43 years) complaining of nasal obstruction and those previously underwent maxillofacial imaging with at least one of the following cross-sectional imaging modalities (computerized tomography, CT $(\mathrm{n}=43)$; magnetic resonance imaging, MRI ( $\mathrm{n}=21)$; positron emmision tomography, PET/CT $(\mathrm{n}=2))$ between January 2005 and May 2010 were recruited. 


\section{Standard Protocol That Was Applied to All Cases Investigated for Nasal Cavity Masses}

In two cases, examinations had been performed with PET/CT device (Discovery ST PET/16 slice CT fusion system HPOWER 60; General Electric Medical Systems, Milwaukee, WI), after intravenous administration of 13 $15 \mathrm{mCi}$ FDG (Fludeoxyglucose) 40 - 60 minutes before the procedure. A section thickness of $3.75 \mathrm{~mm}$ was adjusted for PET images and MPR, MIP and fused images were simultaneously evaluated on multi-display workstations. For fused CT, a section thickness of $3.75 \mathrm{~cm}$, pitch 1.75 , colimation $10 \mathrm{~mm}, \mathrm{kV} \mathrm{120}$, and $\mathrm{mAs} 100-120$ were preferred.

MR imaging had been performed on a 1.5-T scanner (The New Intera Nova Philips Medical Systems, Best, The Netherlands) with variable TR and TE values. Multiplanar, turbo spin-echo and gradient echo mixed images and coronal T2-weighted images were obtained. The section thickness was $5 \mathrm{~mm}$, with an intersection gap of 1 $\mathrm{mm}$. In all cases $(\mathrm{n}=21)$, T1 weighted (coronal-saggital and axial) images enhanced with gadolinium diethylenetniaminepentaacetic acid $(10 \mathrm{ml})$ were obtained.

CT examinations had been performed with 16 slice system (Philips Medical Systems MX 8000 IDT Multislice CT System-V 2.5) in 34 cases or with 64 slice system (Siemens 64 slice CT, Leonardo Running Space) in 9 cases. During all CT examinations, transverse slices of 2 $\mathrm{mm}$ in thickness were obtained from the top of the frontal sinus to the base of the maxillary sinuses. Then, raw data was reconstracted to $1 \mathrm{~mm}$ section thickness. IV contrast material (ioheksol, 300/100, $1.5 \mathrm{mg} / \mathrm{kg}$ ) was administered in 7 patients and all images were also documented using both soft-tissue and skeletal window setting.

All cases that were included in the study had pathologic lesions arising from the nasal septum, nasal passage walls, conchae or the paranasal sinuses, which invaded or obstructed the nasal passage. Common variations such as simple concha bullosa, paranasal sinus derived simple retention cysts, allergic rhinitis, nonfungal rhinosinusitis or nasal polyposis were excluded.

\section{Results}

Benign cases $(n=48)$ consisted of nasal alar hemangioma $(\mathrm{n}=1)$, nasal angiofibroma $(\mathrm{n}=1)$, columellar dermoid cyst $(\mathrm{n}=1)$, fibrous dysplasia of the maxillary sinus $(\mathrm{n}=$ 5), pleomorphic adenoma $(\mathrm{n}=1)$, antrochoanal polyp $(\mathrm{n}=$ 4), sphenochoanal polyp $(\mathrm{n}=1)$, inverted papilloma $(\mathrm{n}=$ $6)$, complicated concha bullosa $(n=6)$, rhinolith $(n=4)$, mucocele $(\mathrm{n}=6)$, atypical retention cyst $(\mathrm{n}=4)$, fungal destructive sinusitis $(\mathrm{n}=3)$, nasoethmoid encephalocele $(\mathrm{n}=4)$, nasal paraganglioma $(\mathrm{n}=1)$. Malignant cases $(\mathrm{n}$ $=18)$ consisted of lethal midline granuloma $(\mathrm{n}=3)$, sinonasal lymphoma $(\mathrm{n}=5)$, chondrosarcoma $(\mathrm{n}=2)$, squamous cell carcinoma $(n=4)$, melanoma $(n=1)$, adenoid cystic carcinoma $(n=1)$, esthesioneuroblastoma $(\mathrm{n}=1)$, sinonazal Ewing sarcoma $(\mathrm{n}=1)$. Our study group was consisted of many lesions which includes many of the reported nasal cavity masses (Table 1).

All cases had varying degrees of nasal obstruction. In

Table 1. A detailed practical classification scheme for the nasal cavity masses.

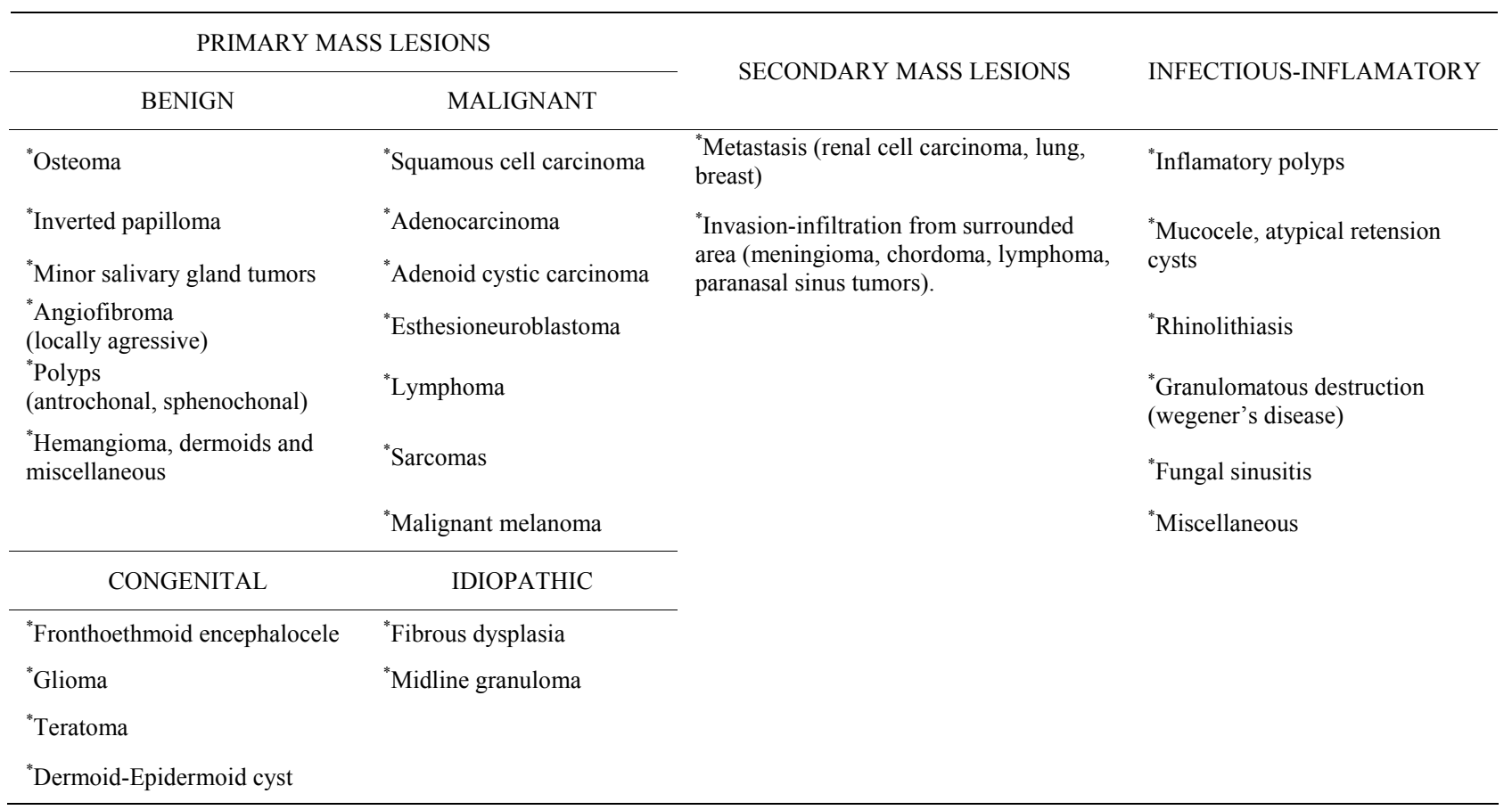


fully ossified lesions (rhinolitis, osteoma, fibrous dysplasia) diffuse hypointensity on MRI and sclerosis on CT was accepted as benign. Smoothly bordered nonenhanced lesions (cysts, secretions, mucoceles) were easily defined in both CT and MRI but better characterized with enhanced MRI images. On the other hand masses with smooth borders but enhanced differently from mucoceles or retention cysts were diagnosed as nerve tissue (Schwannoma, Paraganglioma) or minor salivary gland (pleomorphic adenoma) tumors. Uniform contrast enhancement was associated with hemangioma or angiofibroma. Necrosis, invasion or destruction were associated with malignant neoplasms, granulomatous reactions, fungal infections or metastases. Identification of lipomatous content on cross-sectional images was associated with dermoidepidernoid lesions. Other lesions that associated with cranial vault (fronthoethmoid encephalocele, estesioneuroblastoma, chordoma, meningioma) were easily diagnosed by typical appearences (Scheme I).

\section{Discussion}

Prolonged irritant dust inhalation, smoking, nickel, chrome, radium, isopropyl alcohol, toxic gases such as the mustard gas constitutes the etiology of the nasal cavity tumors. Throughout life, radiation exposure (diagnostic or therapeutic), immunosuppression or lesions that carry the risk for malignant degeneration such as the inverted papilloma increases the frequency of the malignant lesions $[2,3]$. However, nasal cavity malignant lesions are rare, but the similar clinical features of the benign and malignant lesions in the beginning may delay the diagnosis [4].

Inflammatory diseases constitutes another group for differential diagnosis; destruction and erosions of the organ mimic the malignant lesions and may be misdiagnosed [4, 5]. The lesion, benign, malignant or inflammatory must be accurately and quickly diagnosed within these complexity and the final biopsy must address the lesion [4,5]. Especially cases with recurrent, prolonged, unilateral blockage despite the medical therapy and diplopia, proptosis, cranial nevre paralysis must receive paranasal cross-sectional scans [6]. Although the clinical symptoms help to identify a lesion to some extent, it is hard to describe the localization, configuration or the possible invasion of the lesion. If suspected, cross-sectional imaging of the nasal cavity and adjunctive anatomical boundaries must be performed.

In the nasal cavity lesions; detailed examinations of the paranasal sinuses, orbita, intracranial fossa, pterygomaxillary and pterygopalatine fossae and the infratemporal cavity must be made. Adjunctive anatomical invasions both determine the therapeutical approaches and give an idea of the rate of the morbidity and mortality [3,4].

These wide-range nasal cavity/paranasal sinus derived tumors are approximately $70 \%$ benign and $30 \%$ malignant [1]. In a classification including also our cases, it is shown that tumors arising from this region or lesions mimicking the tumors are not so rare (Table 1).

Among these lesions, the most frequent malignant lesion is the squamous cell carcinoma and the most benign lesion is the osteoma. Imaging characteristics, in order as shown in the table are;

- Osteoma and similar sclerotic dense lesions in the $\mathrm{X}$-ray; first a radiography then the $\mathrm{CT}$ provides detailed information about the contour, localization and the nature of the lesion other than the MRI [7].

- Inverted papilloma is unilateral and arises from the lateral nasal wall. Malignant transformation risk is about $2 \%-15 \%$. CT provides information about the oseous changes, remodelling more than destruction and the slowly growing nature of the lesion. Despite the internal contrast in the MRI, differential diagnosis can be made with the intermediate signals other than the high T2W signals of the malign lesions [8]. If needed, both imaging modalities could be used together.

- Choanal polyps could be identified as the lesions originated from the sphenoid or maxillary ostiums and widen in the nasal cavity, thus narrowing the nasal passage. Both CT and MRI could be used.

- Pleomorphic adenoma is a benign mixed tumor of the salivary glands. Although it arises mainly in the major salivary glands, pleomorphic adenoma arising within the nasal cavity has rarely been reported in the literature [9]. Differential diagnosis is hard, but the puctuate calcification in the CT is specific for the lesion. Our case also had puctuate calcifications. These nasal cavity derived well defined lesions may be identified in the MRI, despite the heterogen contrast enhancement, the T2W sequency may show the hypointense capsule surrounding the lesion [9].

- Angiofibroma originates from the sphenopalatine foramen and involves both the pterygopalatine fossa and the posterior nasal cavity [10]. In this lesion no evident destruction is observed through the air plans. Both MRI and CT shows diffuse-homogen and dense contrast enhancement without any necrotic or cystic degeneration and is patognomical [10]. Another highly vascularized benign lesion is hemangioma and involvement of the nasal cavity is exceedingly rare. Cross-sectional images may show masroscopic honeycomb appearance [11].

- Encephalocele, glioma and dermoid cyst are the most common midline (congenital) nasal masses. Given their potential for intracranial extension, prompt treatment is necessary to prevent complications. Although CT is used in this group, the lipomatous content of the lesion or the neural-parenchymal connections could be viewed under the high resolution spin-echo $\mathrm{T} 1 \mathrm{~W}$ and T2W coronal plans [1,12]. 
One of our cases was lipomatous and the dermoid lesion content was measured as -123 Hounsfield Unit on CT images. The other two benign midline lesions could only be diagnosed with the MRI. The term "nasal glioma" is often misleading; it has long been recognized that they are not neoplasms but malformations, and the term "heterotopia" has been referred [13].

Malignant nasal cavity tumors are $80 \%$ squamous cell carcinoma and often originates from the maxillary sinus $(25 \%-58 \%) .10 \%$ of the malignant tumors are adenocarcinoma and adenoid cystic carcinoma. Other malignant tumors are extremely rare. Before they fill the nasal cavity, they mimic the symptoms of the chronic sinusitis, thus once diagnosed, they invade or infiltrate other structures [1]. Local osteolytic, perineural or perivascural invasion or metastatic invasions to the lungs or skeleton identified cross-sectionally addresses the malignant potential of the tumor. Such lesions must be thoroughly evaluated with thin section multi-planar images which obtained by reconstruction in bony-tissue algorithm. Such images permit to detailed evaluation of the cribriform plates, fovea ethmoidalis, planum sphenoidale, posterior frontal sinus wall, and the medial orbital roof. Once identified; malignant tumors of the nasal cavity are highly cellular tumors with little free water, and they may contain focal areas of hemorrhage or necrosis. This is reflected in their heterogenous MR imaging appearances and a low-to-intermediate signal intensity on both $\mathrm{T} 1$ and T2-weighted images contrary to benign ones [14].

Extranodal lymphoid tissue derived lymphomas are in the malignant group that constitute less than $1 \%$ of the nasal cavity tumors. They are often seen in the maxillary or ethmoid sinuses $[15,16]$. They are often non-Hodgkin lymphomas, mostly B cell lymphomas. Despite this group, less aggressive $\mathrm{T} / \mathrm{NK}$ cell lymphomas are rare but their prognosis are worse. Advances in immunocytochemical phenotyping have revealed that the majority of these lesions are in fact either a form of non-Hodgkin's lymphoma arising in the sinonasal tract $[15,16]$. Terms used to refer to such lesions include Stewart's syndrome, lethal midline granuloma, idiopathic midline granuloma, idiopathic midline destructive disease, midline nonhealing granuloma etc. [17]. These older terms have been replaced in the pathologic diagnostic nomenclature of sinonasal disease by new terminology that accurately describes the cellular lineage and biological growth rate [17]. And several attempts have been made to distinguish the various types of midline destructive processes on the basis of imaging findings. Imaging features of this group, different than the other primary malignant nasal cavity masses, are extensive destruction of thye osseous anatomy that may lead to autorhinectomy on the CT images. Our cases also had massive destruction that nearly erased the anatomical structures. Once suspected, serological and histopathological verification must be made at once. Only the cross-sectional images may not be able to differ these lesions from the sarcoma or Wegener granulomatosis [17].

Primary nasal cavity originated malignant melanomas are neural crest tumors that constitutes less than $1 \%$ of all malignant melanomas. They arise mostly from the nasal septum and turbinates. Melanoma must be suspected if a mass is identified from the septum or a pathological focal activity is observed in the nasal cavity during the irrelevant whole body PET/CT scans. Differential diagnosis can be made with MRI, by defining the hyperintensity of the melanin pigment cantaining tumoral tissue [18]. We accidentally determined the nasal dense FDG activity during the PET/CT scan. It is also noted that, as all malignant or inflammatory pathologies of the body, this method is being used more frequently and proven effective $[19,20]$.

Esthesioneuroblastoma (also called olfactory neuroblasto$\mathrm{ma}$ ) is a rare cancer which develops in nerve tissue associated with the sense of smell. When diagnosed in its early stages, esthesioneuroblastoma can often be treated successfully with surgery, radiation and/or chemotherapy (19). On CT and MR sections, the lesion is often centered near the cribriform plate. CT is useful for defining bone destruction, whereas MR imaging best delineates soft-tissue extension. In this context, the use of both modalities is critical for discriminating between postobstructive secretions and tumor tissue, as well as for defining intracranial extension [19,21].

Focal osseous destructive masses of the nasal cavity or paranasal sinuses that also have a soft tissue component may be a sign for metastatic lesions. Generally a lesion of this kind might be renal cell carcinoma, lung or breast carcinoma metastasis and it is hard to differ from the midline aggressive tumors. However, if suspected, the PET/CT scans may identify the primary focus and differentiate the lesion [1].

Benign lesions, although beyond the scope of this study, will be briefly described as they have their part in our algoritm. For example, the nasal polyps have both the chronic inflammation and resorption of the sinunasal structures, so the loss of the marginal sharpness is frequent. Internal linear septal contrast enhancement is patognomic in the postcontrast cross-sectional scans [4]. An important point for his group of patients is to prefer MRI for the frequent need for the control scans in order to minimize the amount of the ionized radiation [4]. Retention cysts and mucoceles may have different characteristics of signals in the CT or MRI up to their content and are not typical [4].

Today in the sinunasal pathologies, after the clinical examination, if further examination is needed, excluding the malignancies and detecting distant metastasis, con- 
trast enhanced CT or MRI or PET/CT is the preferred technique. Soft tissue is better visualised by the MRI where on the bony areas CT has more advantagious. Additionally, PET/CT adds functional status of a lesion semiquantitatively allowing to measure the tumor glucose consumption. Thus, these cross-sectional methods can be used tandemly. Especially invasion of the orbital roof, cribriform plate, fovea ethmoidalis, posterior maxillary sinüs wall, pterygopalatine fossa, erosion of the sphenoid sinus wall represent the locally aggressive nature and extranasal invasion of the tumor. The artefact free scans could be better enhanced by the multidetector CT rather than the conventional spiral ones [13]. On the other hand, usually the ostiums of the sinuses are blocked in the sinunasal tumors, so superimposing sinusitis, inflammatory soft tissue deposits and the retention cysts may not provide evident differentiation in the density in $\mathrm{CT}$, they may only be detected by the contrast enhanced multiplanar MRI. In this context, MRI can also differentiate the tumoral tissue from the surrounding edema, fluid or inflammation. Generally the sinunasal tumors have intermediate intensity in the $\mathrm{T} 1 \mathrm{w}$ and $\mathrm{T} 2 \mathrm{w}$ sequences, thefore even without contrast, $\mathrm{T} 2 \mathrm{w}$ hyperintensity of the edema and inflammation could be identified. Intracranial extensions, especially the dural ones, can better viewed by the contrast enhancement MRI examinations [13]. If suspected, PET/CT could be used to measure both the metobolic activity and the aggressive nature of the lesion; besides it could be used to control the response to the treatment [22].

In summary, the mainstay of the radiologic examination in the tumoral cases of the sinunasal cavity is far more than to make a differential histopathologic diagnosis but to explore the origin, dimensions, orientation of the mass to the airway passage and nasal walls, contours and the contrast enhancement of the tumor. Therefore the surgeon may be briefed preoperatively about the nature of the mass and the need for biopsy (also including the guidance) is questioned.

In conclusion, this study gives the cross-sectional hints which might help assess the tumoral specifications, extensions, possible differential diagnoses and the most probable approaches in a practical algoritm that, to best of our knowledge, is the first in the literature (Scheme I).

\section{REFERENCES}

[1] M. F. Mafee, "Nonepithelial Tunlors of Tile Paranasal Sinuses and Nasal Cavity: Role of CT and MR Imaging," Radiologic Clinics of North American, Vol. 31, No. 1, 1993, pp. 75-90.

[2] R. L. Carrau, E. N. Myers and J. T. Johnson, "Paranasal Sinus Carcinoma Diagnosis, Treatment, and Prognosis," Oncology, Vol. 6, No. 1, 1992, pp. 43-50.

[3] J. D. Spiro, K. C. Soo and R. H. Spiro, "Non Squamous
Cell Malignant Neoplasms of the Nasal Cavities and Paranasal Sinuses," Head Neck, Vol. 17, No. 2, 1995, pp. 114-118.

[4] P. M. Som and H. D. Curtin, "Inflammatory Lesions and Tumors of the Nasal Cavities and Paranasal Sinuses with Skull Base Involvement," Neuroimaging Clinics of North American, Vol. 4, No. 3, 1994, pp. 499-513.

[5] M. D. Murphey, E. A. Walker, A. J. Wilson, M. J. Kransdorf, H. T. Temple and F. H. Gannon, "Imaging of Primary Chondrosarcoma: Radiologic-Pathologic Correlation," RadioGraphics, Vol. 23, No. 5, 2003, pp. 1245-1278. doi:10.1148/rg.235035134

[6] S. Safder, J. S. Carpenter, T. D. Roberts and N. Bailey, "The 'Black Turbinate' Sign: An Early MR Imaging Finding of Nasal Mucormycosis," American Journal of Neuroradiology, Vol. 31, No. 4, 2010, pp. 771-774. doi:10.3174/ajnr.A1808

[7] L. Migirov, M. Drendel and Y. P. Talmi, "Osteoma in an Aerated Middle Nasal Turbinate," Israel Medical Association Journal, Vol. 11, No. 2, 2009, p. 120.

[8] A. Dragonetti, R. Gera, A. Sciuto, A. Scotti, A. Bigoni, E. Barbaro and A. Mini, "Sinonasal Inverted Papilloma: 84 Patients Treated by Endoscopy and Proposal for a New Classification," Rhinology, Vol. 49, No. 2, 2011, pp. 207 213.

[9] K. Motoori, S. Yamamoto, T. Ueda, K. Nakano, T. Muto, Y. Nagai, et al., "Inter- and Intratumoral Variability in Magnetic Resonance Imaging of Pleomorphic Adenoma: An Attempt to Interpret the Variable Magnetic Resonance Findings," Journal of Computer Assisted Tomography, Vol. 28, No. 2, 2004, pp. 233-246. doi:10.1097/00004728-200403000-00014

[10] D. J. Howard, G. Lloyd and V. Lund, "Recurrence and Its Avoidance in Juvenile Angiofibroma," Laryngoscope, Vol. 111, No. 9, 2001, pp. 1509-1511.

[11] Y. Noorizan, H. Salina, "Nasal septal haemangioma in pregnancy," Medical Journal of Malaysia, Vol. 65, No. 1, 2010, pp. 70-71.

[12] I. J. Wang, S. L. Lin, K. I. Tsou, M. C. Hsu, W. T. Chiu, S. H. Tsai, et al., "Congenital Midline Nasal Mass: Cases Series and Review of the Literature," Turkish Journal of Pediatrics, Vol. 52, No. 5, 2010, pp. 520-524.

[13] S. M. Allbery, G. Chaljub, N. L. Cho, C. H. Rassekh, S. D. John and F. C. Guinto, "MR Imaging of Nasal Masses," RadioGraphics, Vol. 15, No. 6, 1995, pp. 1311-1327.

[14] R. L. Carrau, E. N. Myers and J. T. Johnson, "Paranasal Sinus Carcinoma Diagnosis, Treatment, and Prognosis," Oncology, Vol. 6, No. 1, 1992, pp. 43-50.

[15] J. D. Spiro, K. C. Soo and R. H. Spiro, "Nonsquamous Cell Malignant Neoplasms of the Nasal Cavities and Paranasal Sinuses," Head Neck, Vol. 17, No. 2, 1995, pp. 114-118.

[16] G. Hartig, K. Montone, M. Wasik, A. Chalian and R. Hayden, "Nasal T-Cell Lymphoma and the Lethal Midline Granuloma Syndrome," Otolaryngology-Head and Neck Surgery, Vol. 114, No. 4, 1996, pp. 653-656. doi:10.1016/S0194-5998(96)70264-4

[17] K. R. Cleary and J. G. Batsakis, "Sinonasal Lymphomas," The Annals of Otology, Rhinology \& Laryngology, Vol. 
103, No. 11, 1994, pp. 911-914.

[18] S. Ciolofan, E. Ioniță, C. A. Mogoantă, F. C. Popescu, F. Anghelina, L. Chiuţu, G. Stanciu, et al., "Malignant Melanoma of Nasal Cavity," Romanian Journal of Morphology and Embryology, Vol. 52, No. 2, 2011, pp. 679-684.

[19] B. D. Nguyen, M. C. Roarke, K. D. Nelson and B. W. Chong, "F-18 FDG PET/CT Staging and Posttherapeutic Assessment of Esthesioneuroblastoma," Clinical Nuclear Medicine, Vol. 31, No. 3, 2006, pp. 172-174. doi:10.1097/01.rlu.0000200735.55296.73

[20] H. Boo and J. P. Hogg, "Nasal Cavity Neoplasms: A Pictorial Review," Current Problems in Diagnostic Radiology, Vol. 39, No. 2, 2010, pp. 54-61.

\section{Appendix}

Scheme I. Hints for diagnosis of a mass which presented by the obliteration of the nasal cavity.

Step by step to the lesion differentiation involving nasal cavity with MRI and CT

- Benign: generally nonenhanced lesions

Rhinolith (mildly dense), fibrous dysplasia (groundglass density) or osteoma (very dense) (Figure 1), retention cyst (nons-calloped osseous borders) or mucocele (scalloped-enhancing rim) with insiccipated secretions (Figure 2);

- Benign aggressive: With associated concomitant diseases like diabetes, immune suppression

Fungal infections (mucormycosis);

- Malignant: Densely scattered or arch like calcific islands throughout the tumoral stroma on CT images

Soft tissue sarcomas including calcification (osteosarcoma, chondrosarcoma) (Figure 3).

Existence of the lipomatous content

- Dermoid (Figure 4), epidermoid lesions, postoperative flap or repositioned graft (history!).

In the nonspecisifc appearence on both the T1w-T2w MRI sequences, examination must be continued after contrast administration.

- Mural enhancement

Sinusitis, mucocele (Figure 2), retention cyst; doi:10.1067/j.cpradiol.2009.07.001

[21] M. Y. Padmanabhan, R. K. Pandey, A. Kumar and V. Tandon, "Oral \& Maxillofacial Manifestations in Pediatric Esthesioneuroblastoma-Report of a Case and Review of Literature," Pediatric Dentistry, Vol. 33, No. 3, 2011, pp. 261-264.

[22] A. Almuhaideb, R. Syed, L. Iordanidou, Z. Saad and J. Bomanji, "Fluorine-18-Fluorodeoxyglucose PET/CT Rare Finding of a Unique Multiorgan Involvement of Wegener's Granulomatosis," The British Journal of Radiology, Vol. 84, No. 1006, 2011, pp. 202-204. doi: $10.1259 / \mathrm{bjr} / 22598605$

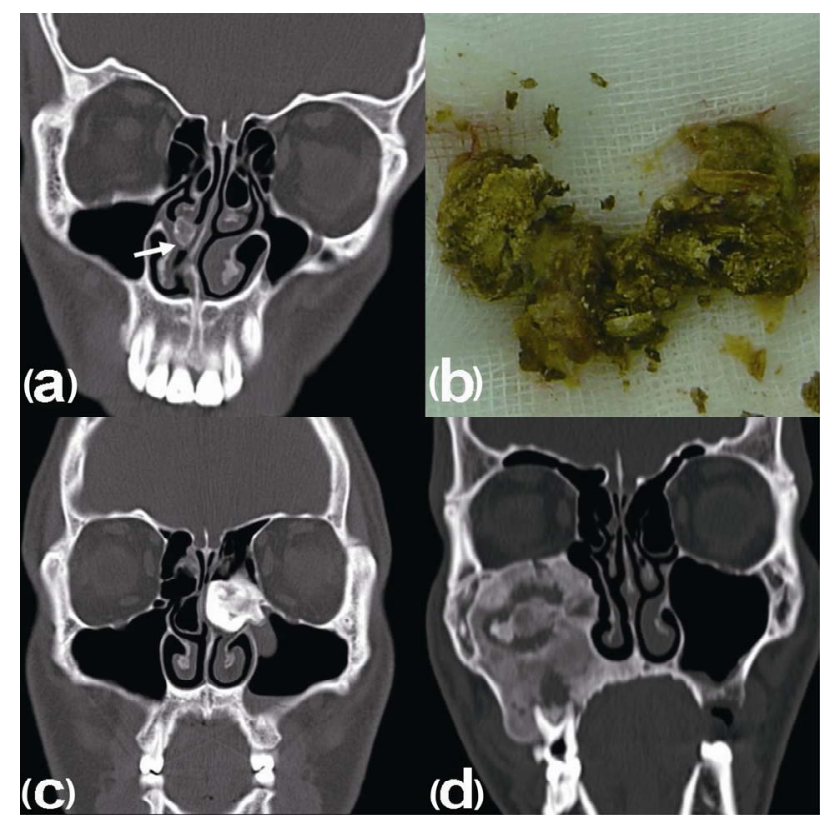

Figure 1. (a) Coronal CT section a case with rhinolith which shows an oval lesion with mild density with relatively lucent central area seated next to the right middle meatus (arrow); (b) After removal it is seen fragmented; (c) A densely sclerosed lesion originated from inferior ethmoid cells and obliterates the left osteomeatal unite on the left side; (d) Diffusely thickened and acalloped borders of right maxillary sinus medial wall also narrows the right nasal passage. 


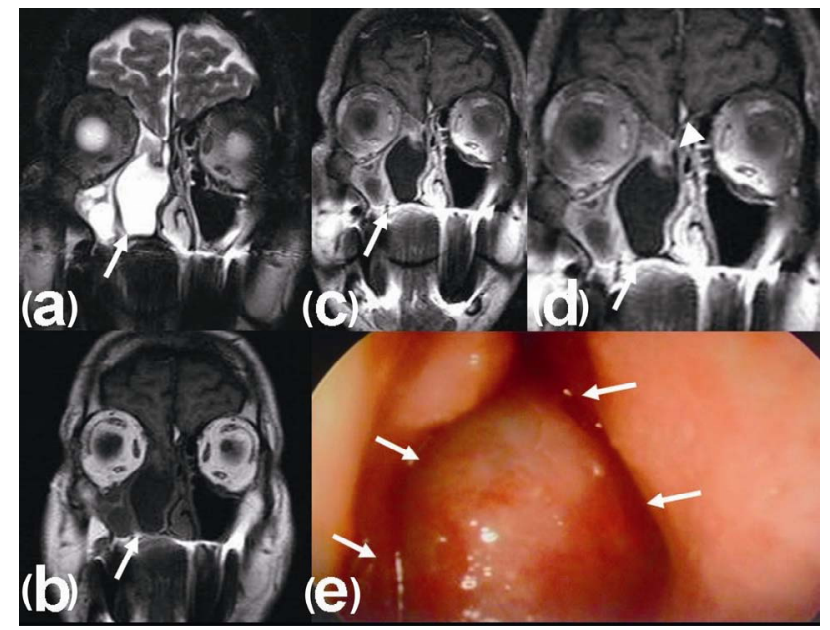

Figure 2. MRI examination of an ethmoid mucocele. (a) Hyperintense on coronal T2w image (arrow); (b) Hypointense on T1w precontrast image (arrow); (c) and (d) On these contrast enhanced coronal fatsaturated images, extention (arrows in (c) and (d)) and origination (arrow in (d)) are seen. Lesion enhanced only murally and showed no internal signal increase on enhanced images. Endoscopic image shows the lesion anphase that emanating from the ethmoid recess.

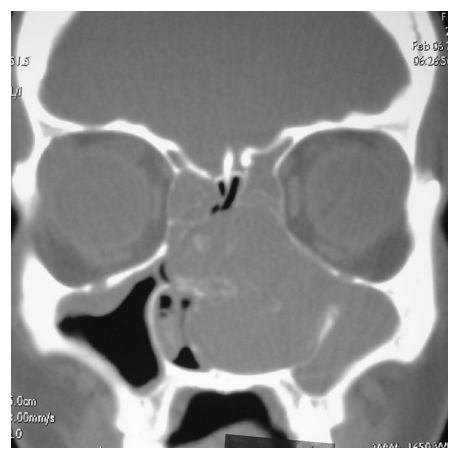

Figure 3. Nasal (left: total, right: partially ) cavity aeration is obliterated by the soft tissue mass that containing scattered calcific islands (chondrosarcoma).

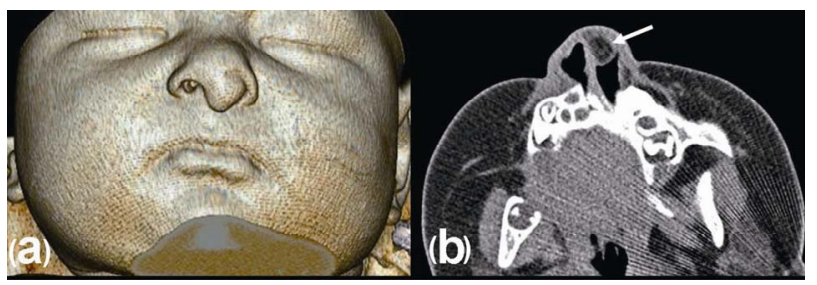

Figure 4. Nasal dermoid lesion. (a) Three-dimentional CT appearence of a columellar lesion; (b) Axial CT image shows the hypodense, lipomatous lesion (arrow).

- Internal septal contrast enhancement

Polipozis (diffuse), polyp (pedinculated with smooth contours), inverting papilloma (pedunculated with lobulated contours, usually originated from osteomeatal unit) (Figure 5);

- Erosion, necrosis or destruction with heterogenous enhancement

Malignant lesions (Squamous cell carcinoma, adenocarcnoma, metastasis), Lymphoma, granulomatous diseases (Figure 6);

- Homogenous and dense contrast enhancement

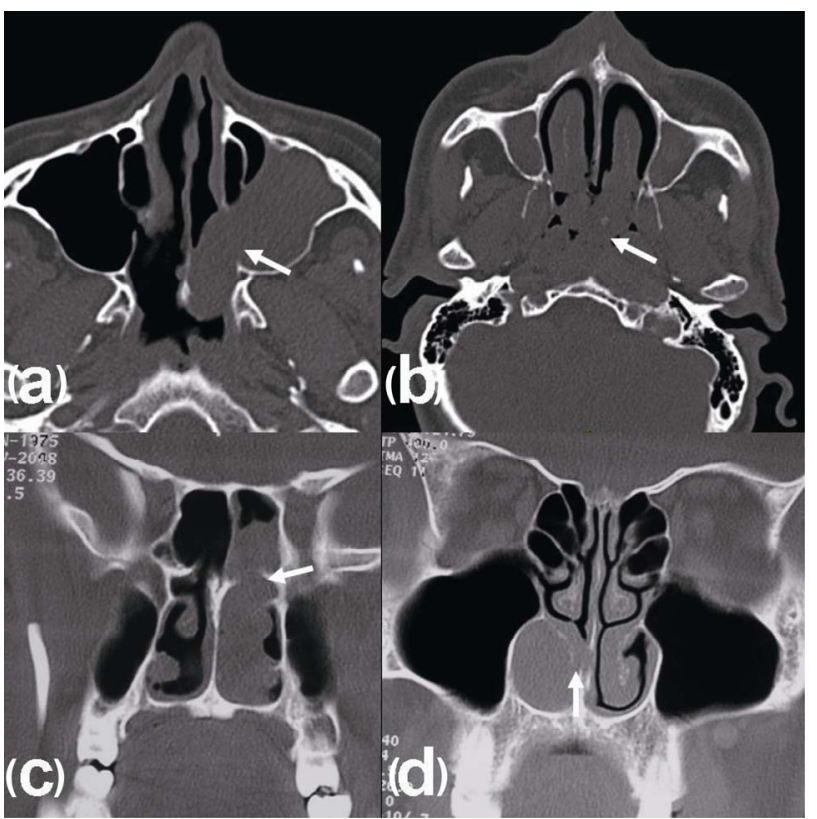

Figure 5. Certain polypoid nasal cavity masses. (a) Antrocoanal polyp, originating from left maksillary sinus antrum (arrow); (b) And extending to the left coanal passage (arrow); (c) A sphenocoanal polyp which originating from the left sphenoid sinus ostium (arrow) with its vertical orientation is diagnostic (arrow); (d) Although having somooth (atypically) borders, erosion of the right inferior turbinate chondrovomeral junction (arrow) direct the diagnosis to inverting papilloma.

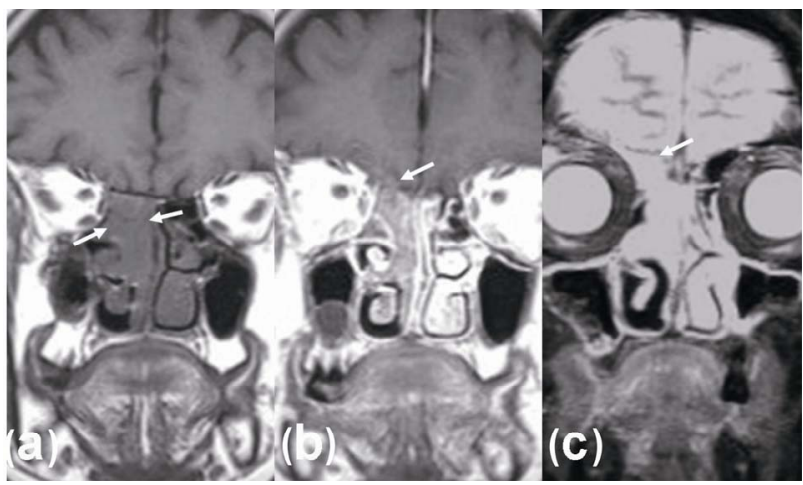

Figure 6. Squamous cell carcinoma. (a) Obliteration of the right nasal passage with a mass that has not obvious borders that seated between right ethmoid cells and nasal cavity (between arrows); (b) Unfortunately, erosion of the right cribriform plate and enhancement with frontobasal focal dural thickening (arrow) shows the cranial involvement; (c) Also, obliteration of the right frontonasal recess causes right frontal sinusitis (arrow). Permits transfer to the dural surface of anterior cranial fossa (b), (arrow). 


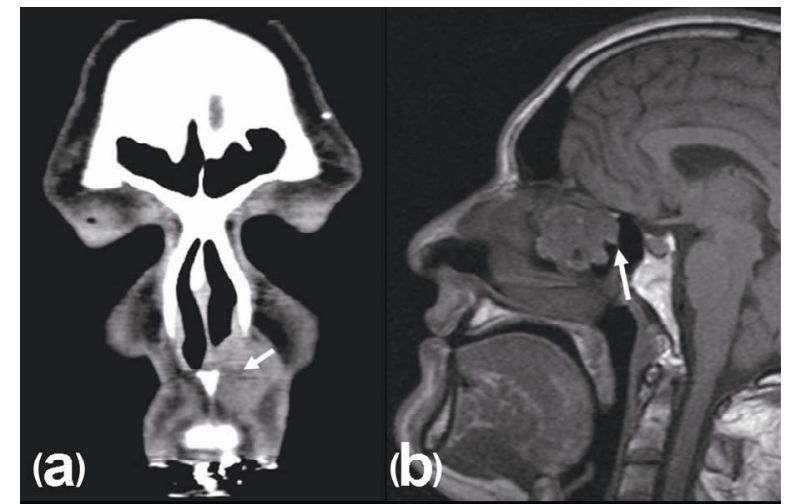

Figure 7. (a) Left nasal alar area located lesion causes partially narrowed aeration and contains some hypodense tubulolineary focuses (arrow), hemangiom; (b) At the posterosuperior pharyngeal location, an expansile mass lesion is seen with scalloped anterior borders of the anterior wall of the sphenoid sinus (arrow), angiofibroma.

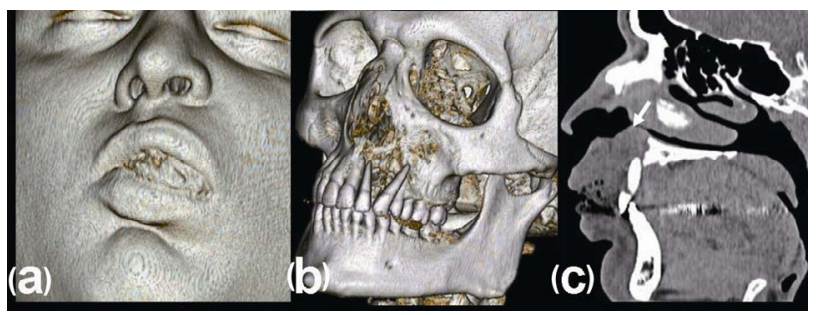

Figure 8. Lymphoma. (a) An expansile nasomaxillary mass causes facial asymmetry at 3D-CT volume rendered image; (b) When the window settings are adjusted to the bone level, it is possible to see the destruction with free teeth; (c) Saggitally reconstructed CT image shows the obliteration of the nasal passage inferiorly (arrow).

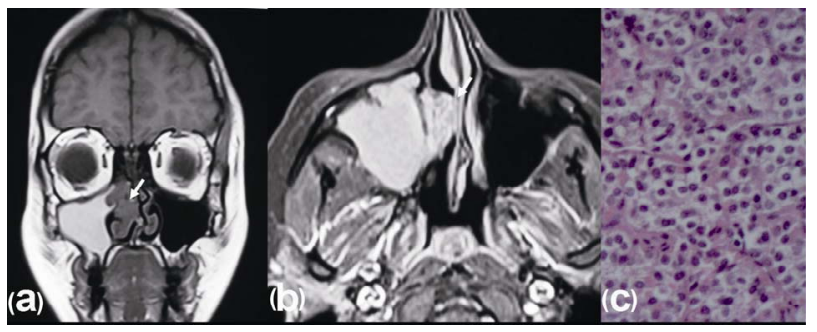

Figure 9. Paraganglioma. (a) A small right nasal mass located in middle meatus, causes accumulation of T1w hyperintense secretion in the right maxillary sinus due to obliteration of the right osteomeatal unit (arrow); (b) On postcontrast axial T1w sequence, lesion enhanced densely and heterogenously; (c) At the cytopathologic examination, it can be seen that tumor is composed of cells which have pale eozinophilic cytoplasm and round nucleus. There is prominent vasculer network separating the tumor nests $(\mathrm{H} \& \mathrm{E} \times 100)$.

Tubulary hypodense or hypointense vasculary traces (hemangioma), Angiofibroma (infiltrative and expansile) (Figure 7).

Pronounced midline destruction (can be diagnosed only with histopathological examination)

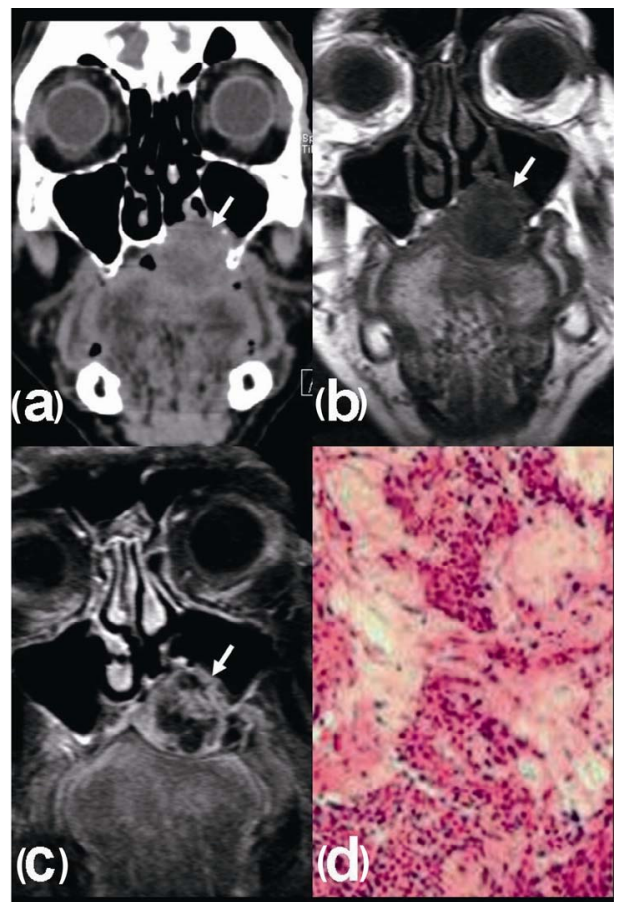

Figure 10. Involvement of the palatum durum with an expansile lesion (paraganglioma) on coronal CT image (a), arrow); And on coronal T1w MRI image (b), aarrow; Postcontrast coronal fatsaturated T1w image shows the heterogenously enhancement of the lesion (c), arrow; The epithelial-myoepitehial islands of tumor in myxoid stroma (d) (H\&E $\times 200)$.

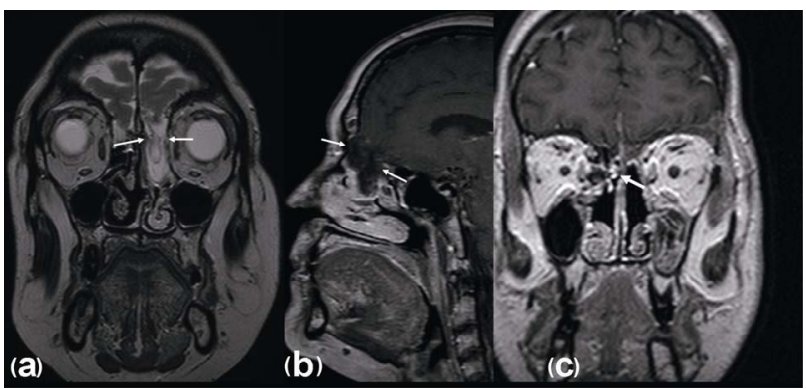

Figure 11. Fronthoethmoid encephalocele. (a) T2w coronal sequence and (b) T1w postcontrast saggital image show the continuity of left fronthobasal neural parenchyma thorough the lef upper and middle nasal meati. This typical appearence must be taken into consideration to prevent any dangerous intervention. Because (c) similiar appearence may be presented in a malignant tumor of this region, esthesioneuroblastoma. Heterogenous tumoral enhancement after contrast injection can differentiate both lesion (arrow).

- Benign

Granulomatous disease (Wegener);

- Malignant

Lymphoma (Figure 8), other primary malignant tumors or metastases.

Masses with smooth borders but enhanced differently from mucoceles or retention cysts 
Nerve tissue (Schwannoma, Paraganglioma) (Figure 9), minor salivary gland (pleomorphic adenoma) (Figure 10).

Cranial Vault Associated (can be diagnosed by typical appearences)

- Benign
Fronthoethmoid encephalocele (a reces which contains basla frontal gyri) (Figure 11);

- Malignant

Estesioneuroblastoma (Figure 11), Meningioma, Chordoma (typical location and appearences). 